Portland State University

PDXScholar

Electrical and Computer Engineering Faculty

Publications and Presentations

Electrical and Computer Engineering

5-1-1975

\title{
Plasma Diagnosis by Laser Beam Scanning
}

Lee W. Casperson

Portland State University

T. K. Cheng

University of California, Los Angeles

Follow this and additional works at: https://pdxscholar.library.pdx.edu/ece_fac

Part of the Electrical and Computer Engineering Commons

Let us know how access to this document benefits you.

\section{Citation Details}

Casperson, L. W., Cheng, T. K. (1975). Plasma diagnosis by laser beam scanning. Journal of Applied Physics. 46 (5), 1961-1965.

This Article is brought to you for free and open access. It has been accepted for inclusion in Electrical and Computer Engineering Faculty Publications and Presentations by an authorized administrator of PDXScholar. Please contact us if we can make this document more accessible: pdxscholar@pdx.edu. 


\title{
Plasma diagnosis by laser beam scanning*
}

\author{
T. K. Cheng and Lee W. Casperson \\ School of Engineering and Applied Science, University of California, Los Angeles, California 90024 \\ (Received 9 September 1974)

\begin{abstract}
A procedure is developed for determining the radial electron density and temperature profiles of a rotationally symmetric plasma. This high-resolution technique involves measuring the deflection of laser beams incident transverse to the plasma. Diagnosis of a stable atmospheric pressure argon arc with $\mathrm{CO}_{2}$ and $\mathrm{He}-\mathrm{Ne}$ laser beams yields results in good agreement with spectroscopic methods.
\end{abstract} \\ PACS numbers: $52.70 . \mathrm{K}$
}

\section{INTRODUCTION}

When a plasma has been created in the laboratory or in nature, it is often desirable to measure some of its properties. Very few lab plasmas behave exactly as predicted by the designer, and the large number of degrees of freedom makes it difficult to work with a really complete theory. Among the plasma parameters of interest are the electron, ion, and atom densities and temperatures, Debye lengths, and correlation properties of the plasma fluctuations. The electron density $N_{e}$ is often the most important parameter, and a number of techniques have been developed for its measurement.

At densities below about $10^{13} \mathrm{~cm}^{-3}$ microwave and probe methods provide useful low-resolution data. For higher densities a variety of optical procedures have been applied. From spectroscopic studies of the continuum and resonance emission lines the electron temperature and density can be determined. ${ }^{1}$ Laser methods include interferometry ${ }^{2}$ and scattering. ${ }^{3}$ Every plasma diagnostic procedure has its own characteristic advantages and disadvantages. Probes, for example, tend to perturb the plasma, have slow time response, and melt at high temperatures. Some interferometers are difficult to set up, susceptible to vibrations, and may yield only the maximum electron density. The purpose of this work is to discuss a laser probing technique based on the deflection of a light beam as it propagates across an axially symmetric plasma. This method is straightforward and does not perturb the plasma. High-resolution plots of the entire radial density profile are obtained.

In Sec. II is discussed the theoretical aspects of our beam deflection technique. Experimental results for a high-density argon arc jet are described in Sec. III. In these experiments $\mathrm{CO}_{2}$ and $\mathrm{He}-\mathrm{Ne}$ lasers are employed, and the maximum electron density observed is $10^{17}$ $\mathrm{cm}^{-3}$. The results agree well with spectroscopic measurements. Related beam deflection measurements were made recently using He-Ne laser beams of wavelengths 3.39 and $1.15 \mu .{ }^{4}$ With these shorter wavelengths the deflection angles are small and the data was only used to obtain the maximum electron density. For densities in the range $10^{12}-10^{14} \mathrm{~cm}^{-3}$ the plasma profile has been studied by means of microwave beam refraction. ${ }^{5,6}$

\section{THEORY}

The diagnostic technique described here depends on the deflection of a laser beam as it passes through a plasma. We first develop relationships betwe en the measured deflection angles and the radial electron density profile. The index of refraction of a plasma has a component due to the free electrons and a component due to the atoms and ions. Both components are wavelength dependent, and their relative importance can be determined if the measurements are made at more than one wavelength.

The indices of refraction of a plasma at the wavelengths $\lambda_{1}$ and $\lambda_{2}$ are given approximately by the expressions ${ }^{7}$

$$
\begin{aligned}
& n_{1}(r)-1=\left(A+B / \lambda_{1}^{2}\right) N_{a}(r)-C \lambda_{1}^{2} N_{e}(r), \\
& n_{2}(r)-1=\left(A+B / \lambda_{2}^{2}\right) N_{a}(r)-C \lambda_{2}^{2} N_{e}(r),
\end{aligned}
$$

where $N_{e}$ is electron density and $N_{a}$ is the effective atomic density. The coefficients $A$ and $B$ depend on the type of plasma. For an argon discharge the values are $A=1.039 \times 10^{-23} \mathrm{~cm}^{3}$ and $B=5.82 \times 10^{-34} \mathrm{~cm}^{5}$, and $N_{\text {o }}$ $=N_{\text {at om }}+0.8 N_{\text {1on }} \cdot{ }^{4}$ The coefficient $C$ is a constant equal to $e^{2} / 2 \pi m c^{2}=4.48 \times 10^{-14} \mathrm{~cm}$, where $e$ is the electron charge, $m$ is the electron mass, and $c$ is the velocity of light. At the long wavelength used in our experiment $\left(\lambda_{2}=1.06 \times 10^{-3} \mathrm{~cm}\right)$ the index of refraction is determined mostly by the free electrons, while at the short wavelength used $\left(\lambda_{1}=6.33 \times 10^{-5} \mathrm{~cm}\right)$ the atomic and ionic effects are dominant.

Equations (1) and (2) can be solved for the two unknowns $N_{e}(r)$ and $N_{a}(r)$ and the results are

$$
\begin{aligned}
& N_{e}=\frac{\left(A+B / \lambda_{1}^{2}\right)\left(n_{2}-1\right)-\left(A+B / \lambda_{2}^{2}\right)\left(n_{1}-1\right)}{\left(A+B / \lambda_{2}^{2}\right) C \lambda_{1}^{2}-\left(A+B / \lambda_{1}^{2}\right) C \lambda_{2}^{2}}, \\
& N_{a}=\frac{\lambda_{1}^{2}\left(n_{2}-1\right)-\lambda_{2}^{2}\left(n_{1}-1\right)}{\lambda_{1}^{2}\left(A+B / \lambda_{2}^{2}\right)-\lambda_{2}^{2}\left(A+B / \lambda_{1}^{2}\right)} .
\end{aligned}
$$

Using the coefficient values indicated previously and the wavelengths appropriate to our experiments, Eqs. (3) and (4) reduce to

$$
\begin{aligned}
& N_{e}=-1.99 \times 10^{19}\left(n_{2}-1\right)+1.96 \times 10^{19}\left(n_{1}-1\right), \\
& N_{a}=-3.39 \times 10^{20}\left(n_{2}-1\right)+9.52 \times 10^{22}\left(n_{1}-1\right) .
\end{aligned}
$$

Equations (3) and (4) express the electron and atomic densities in terms of the indices of refraction measured at $\lambda_{1}$ and $\lambda_{2}$. The remaining problem is to relate the indices of refraction to some experimentally measurable quantities. It has been shown that when the index of refraction in the vicinity of a Gaussian laser beam varies sufficiently smoothly the beam remains Gaussian although its diameter, phase front curvature, and direction of propagation may all be functions of position in the medium. ${ }^{8}$ The specific requirement is 


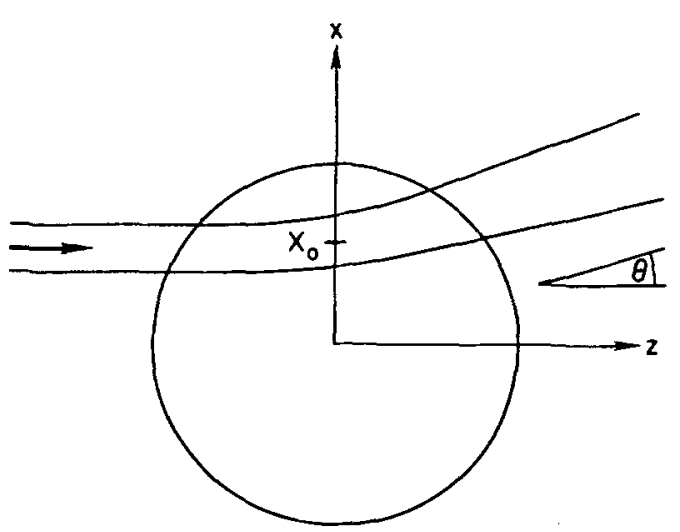

FIG. 1. Schematic drawing showing the interaction of a Gaussian laser beam and a cylindrical plasma.

that the transverse index variations in the vicinity of the beam must be expandable in a Taylor series including at most quadratic terms. If the index of refraction for a $z$ propagating beam is

$$
\begin{aligned}
n(x, y, z)= & n_{0}(z)-\frac{1}{2} n_{1 x}(z) x-\frac{1}{2} n_{1 y}(z) y-\frac{1}{2} n_{2 x}(z) x^{2} \\
& -\frac{1}{2} n_{2 y}(z) y^{2},
\end{aligned}
$$

then the $x$ displacement of the amplitude center of the beam propagates according to the paraxial ray equation

$$
\frac{d}{d z}\left(n_{0} \frac{d x}{d z}\right)=-\frac{1}{2} n_{1 x}-n_{2 x} x=\frac{\partial n}{\partial x} .
$$

The raylike propagation of Gaussian beams has also been verified experimentally. ${ }^{9}$ The spot size $w_{x}$ and phase front curvature $R_{x}$ in the $x$ direction are governed $b^{8}$

$$
Q_{x}^{2}+\frac{2 \pi n_{0}(z)}{\lambda} \frac{d Q_{x}}{d z}+\frac{4 \pi^{2} n_{0}(z) n_{2 x}(z)}{\lambda^{2}}=0,
$$

where $Q_{x}$ is the complex parameter

$$
Q_{x}=\frac{2 \pi n_{0}}{\lambda R_{x}}-\frac{2 i}{w_{x}^{2}}
$$

The situation of greatest interest here involves a Gaussian beam crossing a cylindrically symmetric plasma as shown schematically in Fig. 1. From Eq. (8) the deflection angle $\theta=d x / d z$ shown in Fig. 1 is governed by

$$
\theta\left(x_{0}\right)=\left.\frac{1}{n_{0}} \int_{-\infty}^{\infty} \frac{\partial n}{\partial x}\right|_{x_{0}} d z .
$$

TABLE I. Deflection-angle profiles and assoctated refractivity profiles.

\begin{tabular}{ll}
\hline \hline$\frac{n_{0}}{\alpha} \theta\left(x_{0}\right)$ & $-\frac{n(r)-n_{0}}{\alpha}$ \\
\hline (A) $4\left(x_{0} / R\right)\left[1-\left(x_{0} / R\right)^{2}\right]^{1 / 2}$ & (a) $1-(r / R)^{2}, r<R$ \\
& $0, \quad$ (b) $\left[1+(r / R)^{2}\right]^{-1}$ \\
(B) $\pi\left(x_{0} / R\right)\left[1+\left(x_{0} / R\right)^{2}\right]^{-3 / 2}$ & (c) $\exp \left[-(r / R)^{2}\right]$ \\
(C) $2 \sqrt{\pi}\left(x_{0} / R\right) \exp \left[-\left(x_{0} / R\right)^{2}\right]$ &
\end{tabular}

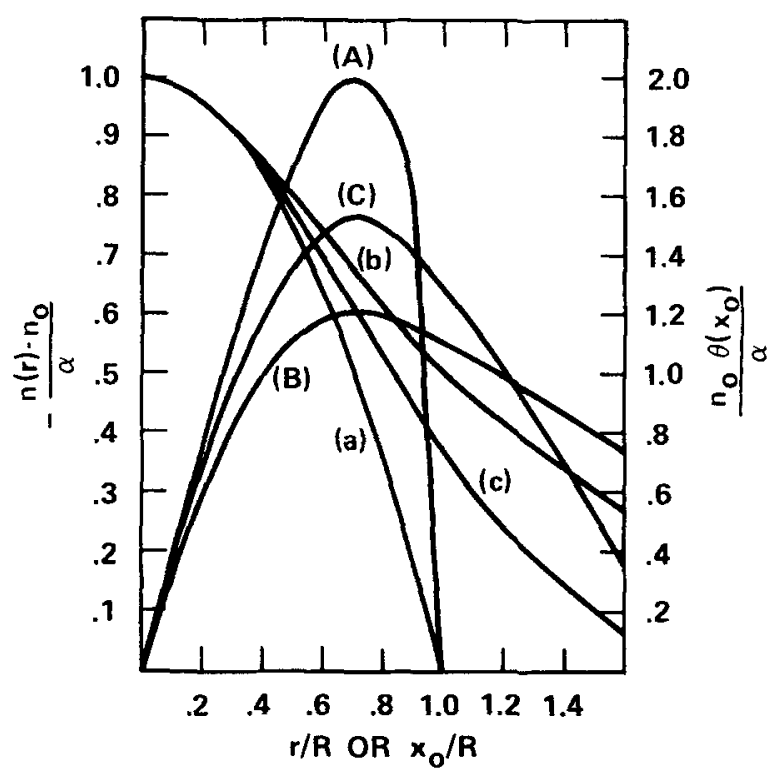

FIG. 2. Deflection-angle profiles and associated refractivity profiles fromTable 1 .

Since the plasma has cylindrical symmetry, the index of refraction can be expressed as a function of the coordinate $r=\left(x^{2}+z^{2}\right)^{1 / 2}$. Thus Eq. (11) reduces to

$$
\theta\left(x_{0}\right)=\frac{x_{0}}{n_{0}} \int_{-\infty}^{\infty} \frac{1}{r} \frac{d n}{d r} d z=\frac{2 x_{0}}{n_{0}} \int_{x_{0}}^{\infty} \frac{(d n / d r) d r}{\left(r^{2}-x_{0}^{2}\right)^{1 / 2}} .
$$

Equation (12) can be put in the form of Abel's integral equation, and hence it can be inverted explicitly. ${ }^{10}$ The result is

$$
n(r)-n_{0}=-\frac{n_{0}}{\pi} \int_{r}^{\infty} \frac{\theta\left(x_{0}\right) d x_{0}}{\left(x_{0}^{2}-r^{2}\right)^{1 / 2}} .
$$

Therefore, the radial profile of the refractive index may be determined by measuring the deflection of a laser beam as it is scanned across a plasma and substituting the results in $\mathbf{E q}$. (13). The refraction profile could also be determined by observing the changes in the beam diameter and phase front curvature and using $\mathrm{Eq}$. (9). The analysis for this case is, however, more difficult.

For some refractive-index functions $n(r)$ the integration in Eq. (12) can be performed analytically. This

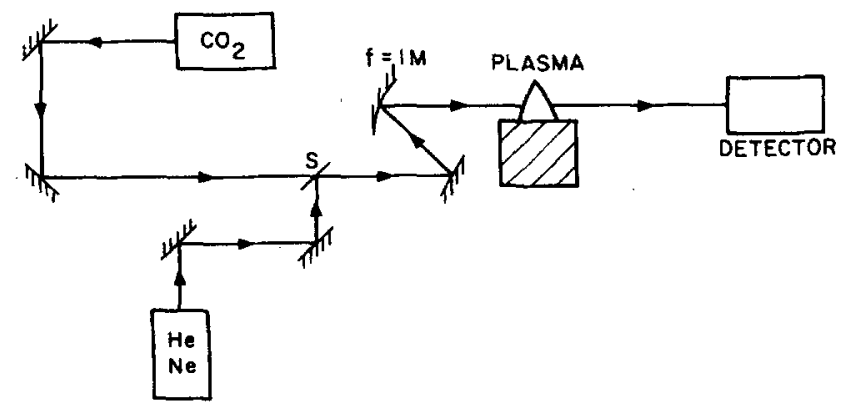

FIG. 3. Experimental setup for beam deflection plasma diagnosis. The flat mirrors immediately following the lasers are for precise beam alignment, and $s$ is a beam splitter. 


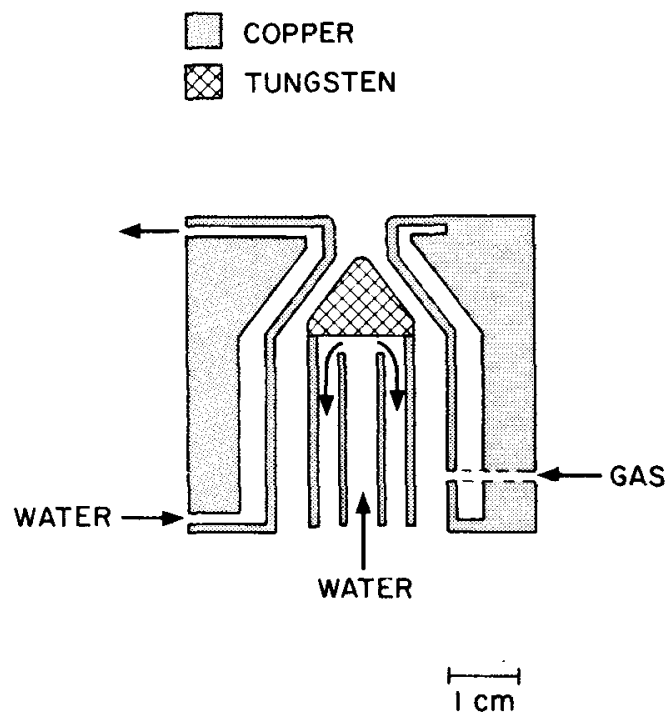

FIG. 4. Scale drawing of the atmospheric pressure argon plasma jet.

is possible for Gaussian, Lorentzian, and parabolic variations, for example, and the results are shown in Table I and Fig. 2 with adjustable constants $\alpha$ and $R$. When the deflection-angle data can be fitted well by a calculated curve, the index-of-refraction profile is known immediately. Otherwise, numerical methods are required.

The numerical analysis of our data has been performed using the Gaussian quadrature approximation. ${ }^{11}$ First the upper limit on the integration in Eq. (13) is replaced by $R$, the radius outside of which there is no refraction of the light beam. Direct numerical integration of the data is not satisfactory because of the singularity at $x_{0}=r$. Therefore, we make the change of variables

$$
s=\frac{x_{0}-r}{R-r}
$$

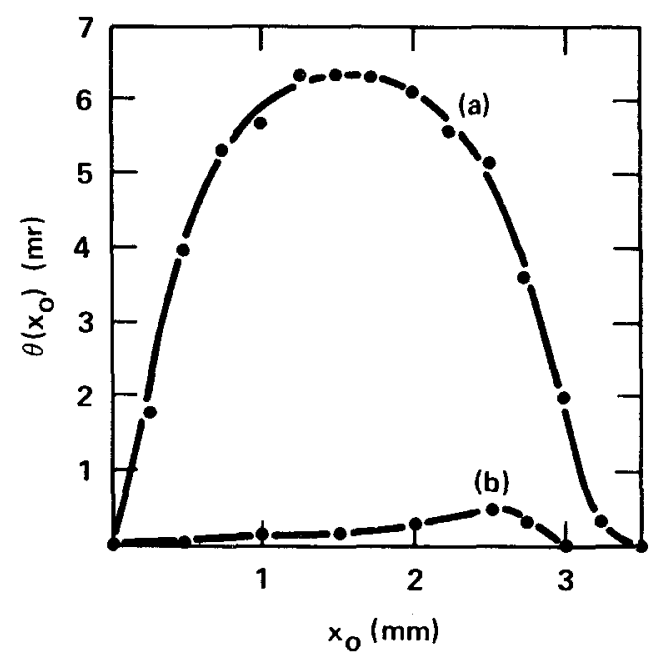

FIG. 5. Experimental deflection-angle data for (a) $10.6-\mu$ wavelength and (b) $0.633-\mu$ wavelength.

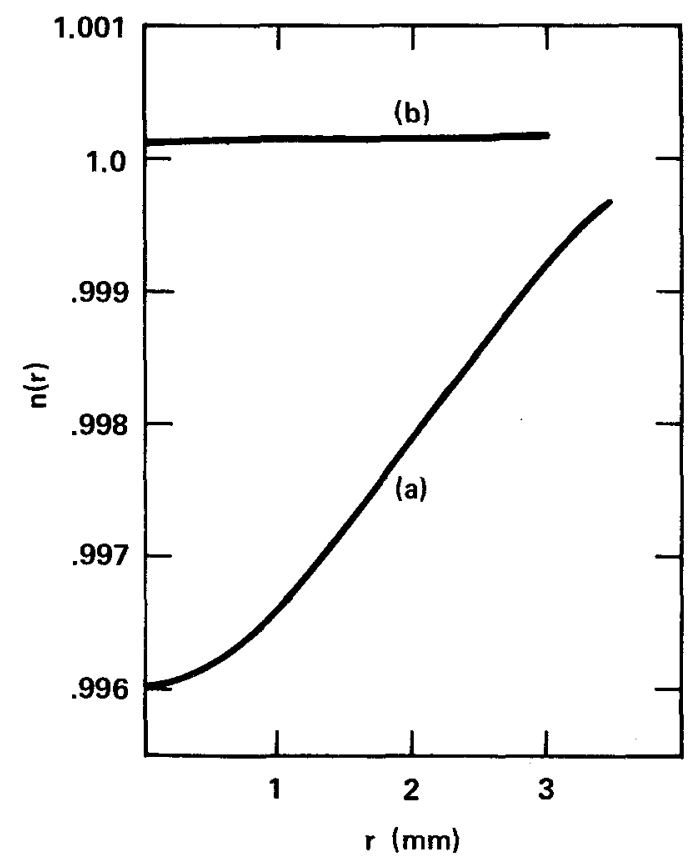

FIG. 6. Refractive-index profiles for (a) $10.6-\mu$ wavelength and $(b) 0.633-\mu$ wavelength.

which leads to

$$
n(r)-n_{0}=\frac{n_{0}}{\pi} \int_{0}^{1} \frac{\theta\left(x_{0}\right)}{s^{1 / 2}}\left(\frac{R-r}{s(R-r)+2 r}\right)^{1 / 2} d s .
$$

Now the result is

$$
\int_{0}^{1} s^{-1 / 2} f(s) d s=\sum_{k=1}^{n} 2 a_{k} f\left(s_{k}\right)+\epsilon,
$$

where $\epsilon$ is a small remainder term and the $s_{k}$ are the squares of the positive roots of the Legendre polynomial $P_{2 n}(y)$, i.e., $s_{k}=y_{k}^{2}(k=1,2, \ldots, n)$. The exact expression for $\epsilon$ is

$$
\epsilon=\frac{2}{4 n+1} \frac{1}{(2 n) !}\left(\frac{2^{2 n}[(2 n) !]^{2}}{(4 n) !}\right)^{2} f^{(2 n)}(s) .
$$

The notation $f^{(2 n)}$ represents the $2 n$ derivative of the function $f$ and $n$ is chosen by repeated differentiation to minimize $\epsilon .^{12}$ For our data analysis the value $n=5$ is chosen, and $\epsilon$ is at most of order $10^{-5}$. Both $s_{k}$ and $a_{k}$ are tabulated for various values of $n .{ }^{11}$ When $n, R$, and $r$ are known, the summation in Eq. (16) can be readily calculated and the radial profile of the electron density then follows from Eq. (3).

\section{EXPERIMENT}

As indicated previously, our refraction experiments involve scanning the Gaussian light beams from $\mathrm{CO}_{2}$ and $\mathrm{He}-\mathrm{Ne}$ lasers across the high-density plasma of an argon arc jet. In addition, spectroscopic techniques have been employed to verify the electron density measurements. The experimental setup is shown in Fig. 3 . First a beam splitter with fine adjustments is used to ensure that the $\mathrm{CO}_{2}$ and $\mathrm{He}-\mathrm{Ne}$ beams are parallel. Then a long-focus spherical mirror $(f=1 \mathrm{~m})$ is used to obtain a minimum $\mathrm{CO}_{2}$ spot size $w_{0}$ of about $0.2 \mathrm{~mm}$ at the plasma. With this configuration the beam is small 


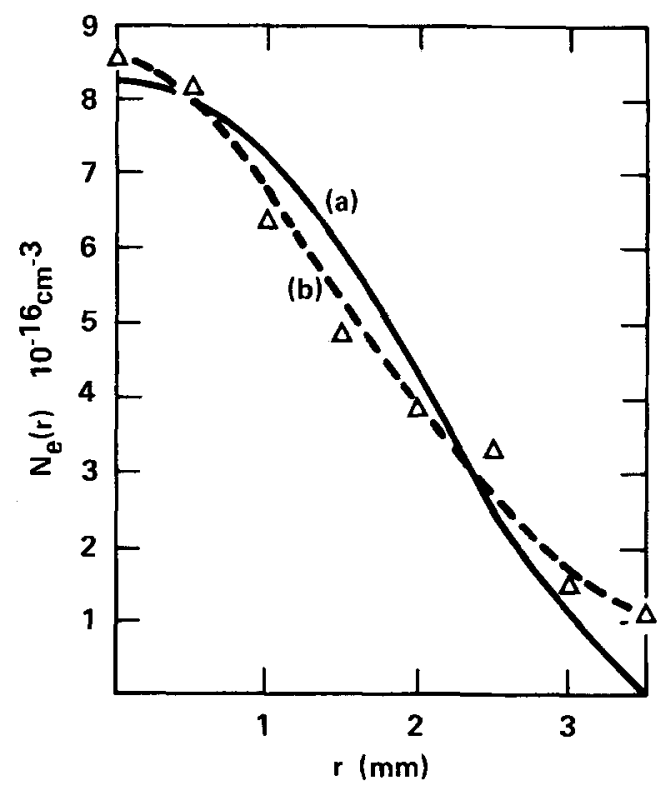

FIG. 7. Electron density profile as determined by (a) beam scanning and (b) spectroscopy.

compared to the plasma diameter, so that the ray equation is valid. Also the deflection angles are usually larger than the Gaussian beam diffraction angle $\theta=\lambda$ / $\pi w_{0}$. The deflection of the intensity maximum of the $\mathrm{CO}_{2}$ beam is determined by scanning a 100- $\AA$ slit attached to a pyroelectric detector at a distance of 2.5 $\mathrm{m}$ from the plasma. For the visible beam a similar procedure is employed using a photomultiplier detector.

The argon plasma jet is shown in detail in Fig. 4 . The tip of the cathode is made from $1 \%$ thoriated tungsten, and the anode is copper. Both the anode and cathode are cooled by flowing water, and the electrode spacing is $2 \mathrm{~mm}$. The dc applied current is 80 to 125 $A$ at $20 \mathrm{~V}$ and the atmospheric pressure argon gas flow is varied between 16 and $50 \mathrm{scfh}$ (standard cubic feet per hour). Under these conditions the plasma is very stable and may be operated continuously for hours.

A typical set of experimental deflection-angle data is shown in Fig. 5. For this experiment the arc current is $100 \mathrm{~A}$ and the argon gas flow is $50 \mathrm{scfh}$. This data has been inverted numerically using $\mathrm{Eq}$. (16) and the resultant index-of-refraction profiles are shown in Fig. 6. Finally, the refractivity data has been combined using Eq. (5), and the electron density profile is plotted in Fig. 7. From these results it is clear that under the high-density conditions used here the refractivity at $0.633 \mu$ is relatively unimportant. Thus the density profile could be simply estimated from a single laser beam scan at $10.6 \mu$ according to $N_{e}(r) \approx-1.99$ $\times 10^{19}\left[n_{2}(r)-1\right]$.

The electron density measurements have been verified by means of a spectroscopic technique. A lens and aperture are used to image a $0.05-\mathrm{mm}$-diam region of the plasma on the entrance slit of a $0.25-\mathrm{m}$ Jarell-Ash monochrometer. The output radiation is detected with a Du Mont type 6467 photomultiplier. The ArI emission lines at $4158.6,4272$, and $4259.4 \AA$ are plotted on an $X-Y$ recorder for various transverse positions across the plasma. With these lines it has been shown by the self-illumination method that self-absorption is unimportant for the conditions of our plasma. The radial intensity distribution has been obtained from the transverse scan data by Abel inversion. ${ }^{13}$ From the radial distribution the temperature can be found by the relative intensity method. ${ }^{14} \mathrm{~A}$ semilog plot of $I / \mathrm{Ag} \nu$ vs $E$ for the three spectral lines yields a straight line where $I$ is the measured line intensity, $A$ is the spontaneous transition probability for the argon lines, $g$ is the statistical weight, $\nu$ is the optical frequency, and $E$ is the excitation energy. ${ }^{15}$ The slope of this straight line is -5040 / $T$ and yields the temperature. Using the known relationship between the electron density and temperature of an atmospheric pressure argon plasma, the electron density profile can be determined. ${ }^{16} \mathrm{~A}$ spectroscopic density profile is given in Fig. 7. The refraction and spectroscopic profiles agree to about $\pm 10 \%$, which is within the errors of the spectroscopic method.

\section{CONCLUSION}

A plasma diagnosis technique has been described which utilizes the deflection of a laser beam as it passes through the refracting plasma medium. The amplitude center of a Gaussian beam propagates as a light ray if the refraction effects are weak, and highresolution absolute density profiles are readily obtained. The technique could be automated using fast scanning high-speed detector arrays and on-line numerical analysis. Applications include many of the plasma configurations of fusion research. The principal laser wavelength is simply chosen shorter than the cutoff wavelength but long enough that substantial electron refraction effects occur. For laboratory plasmas in the range $N_{e}=10^{15}-10^{17} \mathrm{~cm}^{-3}$ the readily available $10.6-\mu$ $\mathrm{CO}_{2}$ laser wavelength is ideal. The validity of this method has been confirmed by comparison with conventional spectroscopic procedures.

\section{ACKNOWLEDGMENT}

The authors wish to acknowledge valuable discussions with Professor M.E. Marhic, Professor D.L. Jassby, and Professor O.M. Stafsudd.

*Work supported in part by the National Science Foundation. ${ }^{1}$ H.R. Griem, Plasma Spectroscopy (McGraw-Hill, New York, 1964), Chaps. 13 and 14.

${ }^{2}$ I. M. Podgornyi, Topics in Plasma Diagnostics (Plenum, New York, 1971), Chap. 7.

${ }^{3}$ E. T. Gerry and D.J.Rose, J. Appl. Phys. 37, 2715 (1966). ${ }^{4}$ P. W. Schreiber and A. M. Hunter II, Plasma Phys. 15, 635 (1973). 
${ }^{5}$ L. A. Dushin, V. I, Kononenko, V. L. Sizonenko, A. I. Skibenko, and K. N. Stepanov, Sov. Phys.-Tech. Phys. 11, 220 (1966).

${ }^{6}$ L. A. Dushin, V. I. Kononenko, and A. I. Skibenko, Sov. Phys, -Tech. Phys. 11, 1372 (1967).

${ }^{7}$ T. J. Morin, Jr. and G. Lanza, Air Force Cambridge Research Lab. Scientific Report No. 1, 1962 (unpublished).

${ }^{8}$ L. W. Casperson, Appl. Opt. 12, 2434 (1973) .

${ }^{9}$ A. N. Rosen, Appl. Opt. 11, 946 (1972).

${ }^{10} \mathrm{H}$.R. Griem, Plasma Spectroscopy (McGraw-Hill, New York, 1964), p. 176.

${ }^{11} \mathrm{~V} . \mathrm{I}$. Krylov, Approximate Calculation of Integrals
(Macmillan, New York, 1962), p. 120.

${ }^{12}$ S. D. Conte, Elementary Numerical Analysis (McGraw-Hill, New York, 1965), p. 85.

${ }^{13}$ K. Bockasten, J. Opt. Soc. Am. 51, 943 (1961).

${ }^{14}$ P.J. Dickerman, Optical Spectrometric Measurements of High Temperatures (Univ. of Chicago Press, Chicago, 1961), p. 129 .

${ }^{15}$ H. N. Olsen, J. Quant. Spectrosc. Radiat. Transfer 3, 59 (1963).

${ }^{16}$ K. S. Drellishak, C.F. Knopp, and A. B. Cambel, Phys. Fluids 6, 1280 (1963). 